\title{
Ecosistema pedagógico y didác- tico policial para la formación de los profesionales de policía de Colombia ${ }^{1}$
}

https://doi.org/10.21830/9789585380202.04

\author{
Onisa Janeth Puche Cabrera ${ }^{2}$ \\ Erika Andrea Camacho Barón ${ }^{3}$ \\ Luis Carlos Cervantes Estrada
}

Escuela de Cadetes de Policía "General Francisco de Paula Santander"

Resumen. La convivencia ciudadana es uno de los pilares que orientan la función policial de la Policía Nacional en los actuales escenarios que enfrenta Colombia y es responsabilidad del mando institucional direccionar sus políticas. El objetivo de este capítulo es establecer los actores que se articulan alrededor de los propósitos formativos de la Universidad Policial, a partir de las necesidades de la institución y en respuesta a las características de los contextos actuales de seguridad y convivencia. Con este propósito se empleó un método de carácter descriptivo-aplicado, con el cual se busca generar conocimiento frente a los componentes asociados a la pedagogía y la didáctica en el contexto policial y su interacción con otros elementos. Se concluye que trascender hacia nuevas

1 Este capítulo forma parte de los resultados del proyecto de investigación "Perfil profesional de Policía del siglo XXI y su ruta de implementación en el proceso de formación inicial”, del grupo de investigación de la Escuela de Cadetes de Policía "General Francisco de Paula Santander", categorizado en B por Minciencias y con código de registro COL0078651. Los puntos de vista que se presentan en este capítulo pertenecen a los autores y no reflejan necesariamente los de las instituciones participantes.

2 Magíster en Educación y especialista en Gerencia Educativa de la Universidad de La Sabana. Especialista en Docencia Universitaria de la Universidad Militar Nueva Granada y especialista en Gerencia de Servicios Sociales de la Universidad Luis Amigó. Profesional en Educación, licenciada en Educación de la Universidad Pedagógica Nacional. Orcid: https://orcid.org/0000-0002-8548-3763 - Contacto: onisa. puche0605@policia.edu.co

3 Magíster en Psicología Clínica, Legal y Forense de la Universidad Complutense de Madrid. Especialista en Investigación Criminal de la Policía Nacional de Colombia. Psicóloga de la Universidad Católica de Colombia. Orcid: https://orcid.org/0000-0003-2061-015 - Contacto: erika.camacho@docentes. umb.edu.co

4 Magíster en Docencia e Investigación Universitaria de la Universidad Sergio Arboleda. Especialista en Investigación Universitaria de la Universidad Sergio Arboleda. Profesional en Lenguas Modernas de la Universidad EAN, Administrador policial de la Dirección Nacional de Escuelas de la Policía Nacional. Orcid: https://orcid.org/0000-0002-5706-3251 - Contacto: luis.cervante@correo. policia.gov.co 
perspectivas pedagógicas redundará, sin lugar a dudas, en profesionales de policía capaces y dispuestos a crear y fortalecer el tejido social, policía-instituciones-comunidad, que contribuya a la construcción de escenarios adecuados para la convivencia en paz.

Palabras clave: coexistencia pacífica; contexto policial; convivencia; didáctica; formación profesional; investigación pedagógica; Policía Nacional.

\section{Introducción}

La convivencia ciudadana es uno de los fines primordiales de la Policía Nacional, según se establece en su misionalidad. Por lo tanto, esta se convierte en uno de los pilares que orientan la función policial en los escenarios actuales que enfrenta Colombia, de manera que es responsabilidad del mando institucional direccionar sus políticas para dar respuesta efectiva a las necesidades de seguridad y convivencia del país en este momento histórico, a fin de lograr "condiciones que permitan la vida en paz, la armonía y la disciplina social" (Londoño, 2013).

En cumplimiento de este objetivo, se planteó una ruta metodológica que incluye un enfoque de corte cualitativo, con un alcance de carácter descriptivo-aplicado. El análisis e interpretación de los resultados se fundamentaron en las siguientes categorías de análisis: (1) cambios y desafíos de la educación policial de cara a las nuevas exigencias de formación de los hombres y las mujeres de la Policía Nacional; (2) capacidades formativas que se deben desarrollar en la educación policial para enfrentar los desafíos emergentes en materia de seguridad y convivencia; (3) líneas de innovación educativa para la consolidación de un ecosistema pedagógico y didáctico policial.

\section{Marco teórico}

La evolución de los cuerpos policiales ha sido una constante debido a la necesidad de adaptación a los cambios sociales, políticos y económicos (Jaschke, 2007), en este sentido, nace la "Visión 2030: Policía, Autoridades y Comunidad - hacia un desarrollo sostenible", con la cual se busca una Policía que soporte su servicio en la convivencia, la seguridad ciudadana y la seguridad 
pública. Desde esta perspectiva, la institución ha orientado sus esfuerzos a la lucha contra el delito, los factores de violencia y los problemas que afectan la seguridad de los ciudadanos, tanto en el ámbito urbano como rural, con un enfoque territorial que contribuya a la acción integrada del Estado. Asimismo, ha direccionado su trabajo a la lucha contra el crimen organizado nacional y transnacional, afectando y desarticulando sus economías ilícitas (Policía Nacional de Colombia, 2018, p. 79).

En este contexto surge el proceso de Modernización y Transformación Institucional (MTI), como mecanismo de fortalecimiento estratégico con el que se busca consolidar un cuerpo de policía más humano, íntegro, disciplinado, innovador y cercano al ciudadano. Lo anterior se traduce en una serie de iniciativas que muestran la hoja de ruta que conduce a lograr las transformaciones institucionales que requiere la Policía Nacional a la luz del posconflicto y frente a la edificación de la paz (Policía Nacional de ColombiaOfpla, 2017).

Es por ello que se presenta el ecosistema pedagógico y didáctico policial, en respuesta a una de las quince líneas estratégicas del proceso de Mтг: "Adaptar la educación policial al nuevo contexto del país", y se considera un desafío institucional demandado por los diferentes sectores de la sociedad. La educación policial se concibe desde un enfoque educativo humano, integral y por competencias, mediante momentos educativos de formación, actualización, entrenamiento y capacitación (Policía Nacional de Colombia, 2013, p. 19).

Desde esta perspectiva, la formación policial es el primer momento educativo que desarrollan sus miembros cuando ingresan a las escuelas de Policía y donde se dimensionan los ámbitos curriculares y didácticos, con el fin de desarrollar las competencias genéricas establecidas por la institución policial para dar cumplimiento a su misión institucional (Resolución No. 1087 de 2019). En este sentido, el objetivo de esta investigación es establecer los actores que se articulan alrededor de los propósitos formativos de la Universidad Policial, a partir de las necesidades de la institución y en respuesta a las características de los contextos actuales de seguridad y convivencia del país. 


\section{Método}

La presente investigación se enmarca en el enfoque cualitativo, con el cual se busca comprender los fenómenos en su ambiente natural a partir de la descripción de situaciones, lugares e individuos (Ramos, 2015, p. 15), con base en la cual se identifican e interpretan fenómenos de acuerdo con los objetos implicados (Blasco \& Pérez, 2007, p. 25). Este enfoque no se basa necesariamente en medición numérica para la interpretación de aquello que se pretende investigar (Sampieri et al., 2014).

Por su orientación, la investigación es de carácter descriptivo-aplicado, ya que busca generar conocimiento frente a los componentes asociados a la pedagogía y la didáctica en el contexto policial y su interacción con otros elementos. Según Hernández et al. (2003), la investigación descriptiva busca especificar propiedades, características y rasgos importantes de cualquier fenómeno que se analice. Por su parte, Vargas (2009) plantea que la investigación aplicada constituye un enlace importante entre la ciencia y la sociedad. Con ella, los conocimientos son devueltos a las áreas de demanda, ubicadas en el contexto donde se da la situación que será intervenida, mejorada o transformada. En este caso, uno de los principales objetivos del estudio es aportar en la adaptación de la educación policial al nuevo contexto de país.

\section{Procedimiento y fases metodológicas}

A continuación se describen las fases de la investigación:

1. Fase 1. Consulta de fuentes documentales. En esta primera fase se pretende identificar y analizar las principales fuentes documentales especializadas que existen sobre el tema de educación policial, pedagogía y didáctica policial y ecosistema.

2. Fase 2. Consulta de fuentes especializadas. La recolección de la información se realizó a través de la entrevista semiestructurada, que permite realizar una caracterización orientada, pero abierta, del proceso de recolección y análisis de los resultados, con la condición de exploración de contenidos y percepciones por parte de los participantes. La entrevista fue validada por medio de una estrategia de 
evaluación de jurados y aplicación del índice de concordancia de Kappa, para establecer los acuerdos formales del instrumento, tales como extensión, coherencia, redacción y pertinencia. A continuación se presenta la caracterización de la población objeto de estudio:

a. Directivos de la Universidad Policial, docentes-directivos y oficiales de planta de las escuelas de Policía.

b. Expertos en educación policial.

c. Mandos del nivel directivo.

d. Estudiantes de los programas de formación de las escuelas de Policía. Para este caso, se utilizó un muestreo intencionado no probabilístico por accesibilidad o conveniencia y se seleccionaron quince actores clave.

3. Fase 3. Estructuración del ecosistema pedagógico y didáctico policial. El tratamiento de los datos recolectados se sistematizó y analizó a través de las siguientes categorías:

a. Cambios y desafíos de la educación policial de cara a las nuevas exigencias de formación de los hombres y las mujeres de la Policía Nacional.

b. Capacidades formativas que se deben desarrollar en la educación policial para enfrentar los desafíos emergentes en materia de seguridad y convivencia.

c. Líneas de innovación educativa para la consolidación de un ecosistema pedagógico y didáctico policial.

\section{Resultados}

A continuación se presentan los resultados de la información obtenida con las técnicas de recolección, con la cual se realizó un análisis descriptivo y exploratorio a través del software estadístico $\mathrm{R}$, que agrupa los conceptos que se usan con mayor frecuencia en una nube de palabras.

Así las cosas, se obtuvieron los siguientes resultados en cuanto a las categorías de análisis: (1) cambios y desafíos de la educación policial de cara a las nuevas exigencias de formación de los hombres y las mujeres de la Policía Nacional; (2) capacidades formativas que se deben desarrollar en la educa- 
ción policial para enfrentar los desafíos emergentes en materia de seguridad y convivencia, y (3) líneas de innovación educativa para la consolidación de un ecosistema pedagógico y didáctico policial:

\section{Cambios y desafíos de la educación policial de cara a las nuevas exigencias de formación de los hombres y las mujeres de la Policía Nacional}

Es claro que en la actualidad la educación superior, en general, enfrenta una serie de cambios vertiginosos debido a las rupturas sociales, económicas, políticas y culturales a las que el mundo se ha visto enfrentado. Como afirma Segrera (2018),

las universidades enfrentan una de las épocas más interesantes, inciertas y complejas, pues la globalización implica la posibilidad de aprovechar oportunidades importantes, pero también desafíos y problemas serios en relación con el futuro [...]. Los valores tradicionales de la universidad siguen siendo válidos (la autonomía, la libertad de cátedra, la investigación, el trabajo de los estudiantes, la evaluación), pero son valores amenazados en el contexto de la globalización. (p. 552)

De igual manera, la incursión de las tecnologías de la información y la comunicación (Tic) en todas las actividades del ser humano hace que las competencias de los nuevos profesionales incorporen, de forma efectiva, modelos digitales para la selección, búsqueda, procesamiento y análisis de la información. En este sentido, Lleixà et al. (2018) afirman que "en el discurso educativo actual, la tecnología se presenta como una herramienta poderosa que ayudará a resolver muchos de los problemas a los que se enfrenta la escuela, al menos en el campo del aprendizaje de conocimientos y habilidades" (p. 14). Lo anterior evidencia que la educación superior debe incorporar en su discurso pedagógico nuevos enfoques educacionales que den respuesta a las exigencias de una sociedad en permanente cambio.

La educación policial no puede estar ajena a esta perspectiva de transformación, toda vez que las variables de la convivencia y la seguridad se reconfiguran día a día, en la cotidianeidad del devenir de las comunidades. Precisamente, es por ello que la Policía Nacional debe analizar concienzudamente nuevas dinámicas de prestación del servicio y de la actividad de policía y percibirlas de una forma diferencial, de tal manera que den respuesta a las 
necesidades y expectativas de los ciudadanos a través de una educación policial prospectiva y conectada con las realidades (figura 1).

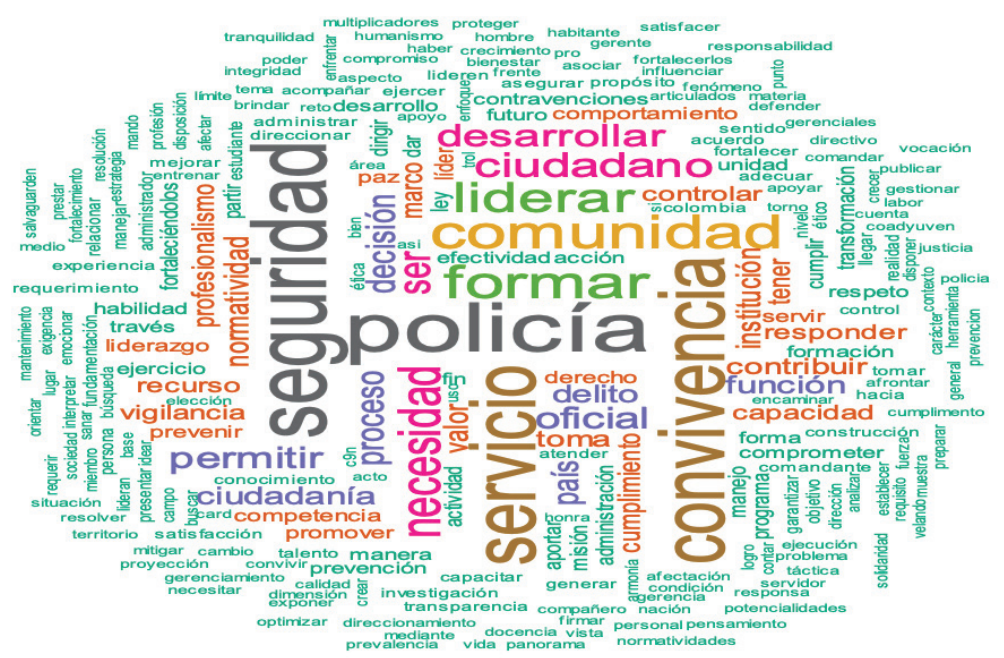

Figura 1. Nube de palabras sobre cambios y desafíos de la educación policial. Fuente: Elaboración propia.

Así las cosas, es claro que existe la necesidad de incorporar nuevas competencias a los procesos de formación de los profesionales de policía. Una de ellas, sin lugar a dudas, es la competencia digital, que va más allá de las habilidades técnicas como la programación o las herramientas de analítica, por ejemplo el uso de estadísticas para medir las tendencias de criminalidad en un contexto específico. Además, es preciso incorporar en los currículos las llamadas competencias blandas, que permiten al policía potenciar su habilidad para interactuar con la comunidad, como el relacionamiento, llegar a las personas y hacerles comprender la razón, el sentido y las consecuencias de las acciones.

Asimismo, el proceso de formación de los futuros profesionales de policía debe focalizar sus esfuerzos en el desarrollo de las demás competencias que los hagan hábiles para enfrentar de la mejor manera los cargos que ocuparán y las responsabilidades que asumirán en la institución. En este sentido, es preciso enseñar la visión estratégica de la institución, como un saber utilizar las herramientas que les da el contexto para la gestión del servicio de policía.

Los entrevistados hacen especial énfasis en el desarrollo de las habilidades blandas de los profesionales de policía, en el entendido que estas competencias, 
también denominadas soft skills, coadyuvarán en la prestación de un servicio de policía más humano. El liderazgo, la comunicación, el trabajo en equipo, la resiliencia, la motivación, la autogestión y la pasión por el servicio son prioritarios en el proceso de formación de los policías.

\section{Capacidades formativas que se deben desarrollar en la educación policial para enfrentar los desafíos emergentes en materia de seguridad y convivencia}

En esta categoría, las respuestas de los participantes coincidieron en tres aspectos importantes: el primer aspecto tiene una inmediata relación con el "ser policía", dado el cambio generacional que se está viviendo en la actualidad en la institución. Formar las nuevas generaciones de policías es un reto pedagógico que incide directamente en los diferentes esquemas educacionales que se han adoptado históricamente en la Policía Nacional. No se trata de cambiar los planes de estudio de los programas académicos, que por demás se encuentran acreditados en alta calidad por el Ministerio de Educación Nacional, sino enfocar la mirada hacia las prácticas pedagógicas desarrolladas.

En esta segunda variable de análisis, los datos recolectados se condensaron en la siguiente nube de palabras (figura 2):

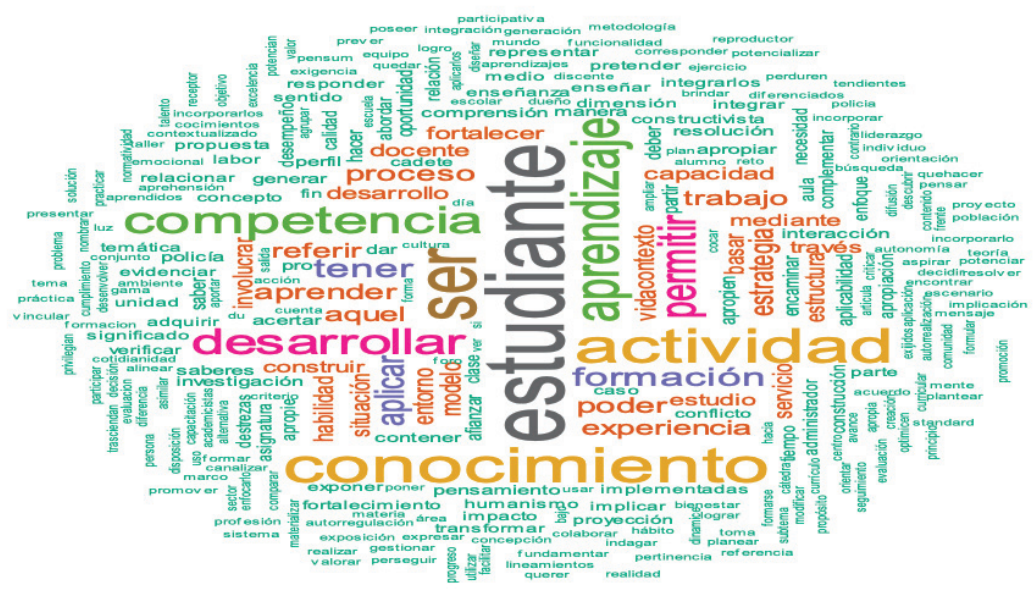

Figura 2. Nube de palabras sobre capacidades formativas en la educación policial. Fuente: Elaboración propia. 
Como se observa, el primer aspecto que se destaca es el "ser" del profesional de policía, que trasciende la órbita de una competencia genérica, para convertirse en el eje gravitacional que facilita el logro de todos los objetivos que la institución se proponga, lo cual justifica su identificación y potenciación a lo largo de toda la carrera policial.

El segundo aspecto está relacionado con la competencia digital que debe permear los propósitos educacionales de los currículos y que permita, por ejemplo, incorporar las metodologías ágiles para el servicio de policía, es decir, la tecnología para facilitar el desarrollo de los procedimientos; incorporar la analítica de los datos para la toma de decisiones acertadas frente a las demandas del ciudadano en cuanto a su seguridad, así como la inteligencia artificial, aspecto que fue planteado en la Política Marco de Convivencia y Seguridad Ciudadana.

Finalmente, el tercer aspecto se refiere a los actores del proceso educativo, ámbito en el que se privilegia a los docentes policiales, toda vez que son ellos quienes se constituyen en la piedra angular del entramado académico. Es aquí donde la calidad de los docentes policiales se constituye en un requisito sine quanon para garantizar la pertinencia de la educación policial, que debe estar a la vanguardia del desarrollo pedagógico y didáctico, así como de los avances tecnológicos que exige ciertas capacidades para su desarrollo. En concordancia con esta perspectiva, la institución está formulando el Modelo de Gestión Docente Policial, con la intención de garantizar profesores que no solo sean expertos en el campo académico-disciplinar, sino que además se identifiquen con la misión y la visión de la Policía Nacional y, sobre todo, que se constituyan en verdaderos formadores de seres humanos.

En este sentido, los docentes son los llamados a fortalecer la filosofía policial, los movilizadores de la cultura institucional, quienes se asumen como actores fundamentales y diferenciales en la co-creación de valor, quienes permiten a largo plazo - pues los resultados educativos no evidencian frutos inmediatos - posicionar a cada integrante de la Policía como generador del cambio social. 


\section{Líneas de innovación educativa para la consolidación de un ecosistema pedagógico y didáctico policial}

Frente a esta categoría de análisis, los entrevistados coincidieron en señalar aspectos gravitantes que permiten consolidar un ecosistema pedagógico y didáctico para la formación de los profesionales de policía de Colombia (figura 3).

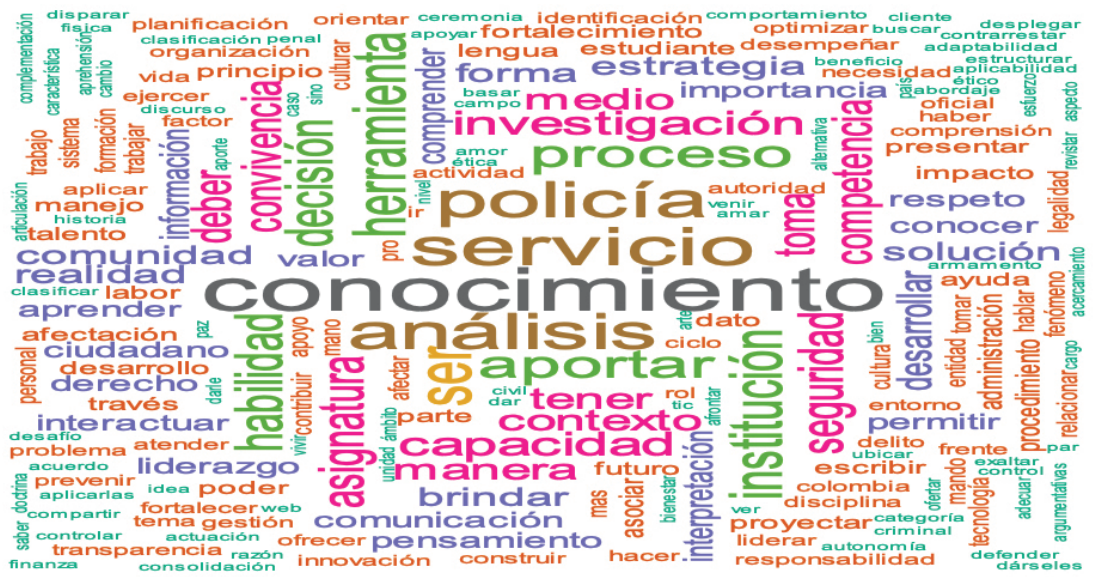

Figura 3. Nube de palabras sobre líneas de innovación educativa policial.

Fuente: Elaboración propia.

La Dirección Nacional de Escuelas como Universidad Policial, con reacreditación en alta calidad otorgada por el Ministerio de Educación Nacional, ha definido como una de sus líneas estratégicas la permanente actualización de sus esquemas educativos, así como la búsqueda constante de estrategias innovadoras que coadyuven en la apasionante tarea de la formación de los hombres y las mujeres policías.

En virtud de lo anterior, la institución adopta un ecosistema pedagógico y didáctico policial que dinamiza los procesos académicos en las escuelas de Policía. Se trata, en primera instancia, de definir el concepto de ecosistema para el entorno educativo policial, entendido como una red de actores (docentes, investigadores, directivos docentes, administrativos docentes, asesores educativos y estudiantes) que se articulan alrededor de los propósitos formativos de la Universidad Policial a partir de las necesidades de la institución, de manera 
que generan y usan el conocimiento policial para responder a las características de los contextos de seguridad y convivencia del país.

$\mathrm{Y}$ es en el marco de este ecosistema que los entrevistados coinciden en el fortalecimiento de tres líneas de innovación educativa: la primera está relacionada con la adopción de una pedagogía policial multimodal, en el entendido de que los nuevos paradigmas educativos no pueden centrarse en un modelo pedagógico único, sino que permiten la incorporación de enfoques que se surten de diferentes teorías y dinámicas; la segunda línea corresponde al campo de la didáctica policial, respecto al cual se promueve que tanto docentes como estudiantes apropien diversas estrategias, técnicas y herramientas de enseñanza y aprendizaje más flexibles y abiertas, de manera que permitan el desarrollo de las competencias digitales; $y$, finalmente, la tercera hace referencia a la implementación de un estilo y un discurso pedagógico situado y contextualizado frente a las nuevas realidades del país en materia de convivencia y seguridad ciudadana. Un discurso que privilegie la práctica sobre la teoría en los espacios de clase, en el entendido que es en el "hacer" que el profesional de policía materializa sus aprendizajes y superar así la brecha de lo aprendido en las escuelas versus la realidad del servicio de policía: "Las prácticas pedagógicas tienen sentido, siempre y cuando se contrasten con la realidad y a su vez respondan tanto a las exigencias que la institución plantea como a las necesidades y perfiles de los estudiantes (Gómez \& Calvo, 2014. p. 5)

Es preciso indicar que, en estas líneas de innovación educativa, la irrupción de las Tic en el campo académico ha marcado un hito histórico trascendental. Es así que para la primera línea enunciada se incorporan las denominadas "pedagogías emergentes", definidas por Adell y Castañeda (2012) como "el conjunto de enfoques e ideas pedagógicas, todavía no bien sistematizadas, que surgen alrededor del uso de las Tic en educación" (p. 15).

Así las cosas, la propuesta es que el modelo pedagógico policial, constructivista con enfoque por competencias, incorpore en sus abordajes otras teorías que le permitan al estudiante-docente transitar de manera más armónica y dinámica, con mayor apertura, sobre el conocimiento y el saber policial.

En cuanto a la segunda línea — generación de prácticas disruptivas de enseńanza policial_-, esta se refiere necesariamente a superar las técnicas que 
tradicionalmente usan los docentes policiales en el aula. Específicamente, en este punto se hace un llamado a que el profesor haga uso del abanico de posibilidades que la era moderna ha traído consigo, así como a que se permita a sí mismo y permita a sus estudiantes explorar otras técnicas y estrategias más libres y abiertas para acceder a nuevos aprendizajes para el quehacer profesional del policía. Al respecto, Christensen (2012; citado por Adell y Castañeda, 2012) indica que "una innovación disruptiva es aquella que crea un nuevo mercado o cadena de valor y destruye la que ha existido durante años o décadas, sustituyendo o desplazando una tecnología anterior" (p. 23).

Lo anterior indica, entonces, que el aula es el escenario propicio para que el docente haga de su práctica de enseñanza un momento de creación y co-creación conjunta y colaborativa, dejando de lado los discursos unidireccionales y, muchas veces, descontextualizados de la realidad del país y del ejercicio de la profesión policial.

Finalmente, este ecosistema pedagógico y didáctico policial favorece la enseñanza y el aprendizaje situado y contextualizado. La intención aquí es que se disminuya la brecha entre las escuelas de Policía y la realidad del ejercicio profesional policial. En este sentido, es fundamental que los estudiantes cuenten con ambientes educativos cercanos a la realidad de las dinámicas cambiantes de la convivencia y la seguridad en el país. Que exista una interacción permanente con el sector externo y el intercambio de experiencias no solo con otras universidades, sino también con diferentes instituciones públicas y privadas con un mismo fin: construir una sociedad más justa, equitativa y en paz.

\section{Conclusiones}

El sistema educativo policial colombiano responde a las exigencias de la comunidad en temas de seguridad ciudadana y convivencia, así como a los lineamientos establecidos por el gobierno nacional en temas de seguridad, educación y ciencia, tecnología e innovación. En este sentido, la Dirección Nacional de Escuelas, como institución de educación superior de la Policía Nacional de Colombia, está llamada a liderar este proceso de reestructuración de la educación policial de cara a los nuevos escenarios de país. 
En este desafío educativo, los nuevos escenarios sociales resultantes de las múltiples dinámicas y transformaciones culturales, políticas y económicas obligan a la educación policial a implementar un ecosistema pedagógico y didáctico, entendido como una red de actores (docentes, investigadores, directivos docentes, administrativos docentes, asesores educativos y estudiantes) que se articulan alrededor de los propósitos formativos de la Universidad Policial a partir de las necesidades de la institución, de manera que generen y usen el conocimiento policial para responder a las características de los contextos de seguridad y convivencia del país.

La actualización permanente de la educación policial en el marco del Proceso de Modernización y Transformación Institucional, como propuesta del Círculo Estratégico de Transformación Institucional (CETIN 5), y los resultados de los análisis aquí planteados, producto de las entrevistas y la revisión documental, determinan la oportunidad, por no decir necesidad, de transformar los modelos educacionales de la Policía Nacional para fortalecer la formación de los hombres y las mujeres de la institución.

Trascender hacia nuevas perspectivas pedagógicas y didácticas redundará, sin lugar a dudas, en profesionales de policía capaces y dispuestos a crear y fortalecer tejido social, policía-instituciones-comunidad, que contribuya a la construcción de escenarios adecuados para la convivencia en paz.

\section{Referencias}

Adell, J., \& Castañeda Quintero, L. J. (2012). Tecnologías emergentes, ¿pedagogías emergentes? https://digitum.um.es/digitum/bitstream/10201/29916/1/Adell_Castaneda_emergentes2012.pdf

Blasco Mira, J. E., \& Pérez Turpin, J. A. (2007). Metodologías de investigación en educación física y deportes: Ampliando horizontes. Editorial Club Universitario.

Gómez, S., \& Calvo, G. (2014). Prácticas pedagógicas exitosas para educar a la Policía de Colombia. http://www.oei.es/congreso2014/memoriactei/1443.pdf

Hernández, R., Fernández C., \& Baptista, P. (2003). Metodología de la investigación (3. ed.). McGraw Hill.

Jaschke, H. E. (2007). Perspectives of police science in Europe. European Police CollegeCEPOL. 
Lleixà, T., Gros, B., Mauri, T., \& Medina, J. L. (Coords.). (2018). Educación 2018-2020: Retos, tendencias y compromisos. Universitat de Barcelona. http://www.ub.edu/ire/ wp-content/uploads/2018/06/IRE-UB_Educacion_2018-2020_CAST.pdf

Londoño, F. (2013). La convivencia, fin del derecho de policía. Magazin ESPOL, (2).

Policía Nacional de Colombia. (2013). Proyecto Pedagógico Institucional: Potenciación del conocimiento. Imprenta Nacional.

Policía Nacional de Colombia. (2018). Plan Estratégico Institucional Comunidades Seguras y en Paz Visión 2030. Policía Nacional de Colombia.

Policía Nacional de Colombia-OFPLA. (2017). Analectas de Doctrina. https://www.policia. gov.co/sites/default/files/publicaciones-institucionales/analectas-doctrina-edicion-04.pdf

Ramos, C. A. (2015). Los paradigmas de la investigación científica. Avances en Psicología, 23(1), 9-17.

Resolución 1087. (2019). "Por la cual se implementan las competencias genéricas en la Policía Nacional". Policía Nacional de Colombia.

Hernández Sampieri, R., Fernández Collado, C., \& Baptista Lucio, P. (2014). Definiciones de los enfoques cuantitativo y cualitativo, sus similitudes y diferencias. En Metodología de la investigación (6. ${ }^{\mathrm{a}}$ ed., pp. 2-21). McGraw Hill Educación.

Segrera, F. L. (2018). Retos de la educación superior en un contexto de incertidumbre y crisis global. Avaliação: Revista da Avaliação da Educação Superior, 23(2), 551-566.

Vargas, Z. R. (2009). La investigación aplicada: una forma de conocer las realidades con evidencia científica. Revista Educación, 33(1), 155-165. 